\title{
Article \\ Digital Twin-Enabled Decision Support Services in Industrial Ecosystems
}

\author{
Jürg Meierhofer ${ }^{1, *(\mathbb{D})}$, Lukas Schweiger ${ }^{1} \mathbb{D}$, Jinzhi Lu ${ }^{2, * \mathbb{D}}$, Simon Züst ${ }^{3} \mathbb{D}$, Shaun West ${ }^{3} \mathbb{D}$, Oliver Stoll $^{3} \mathbb{D}$ \\ and Dimitris Kiritsis 2 (D) \\ 1 School of Engineering, ZHAW Zurich University of Applied Sciences, 8400 Winterthur, Switzerland; \\ lukas.schweiger@zhaw.ch \\ 2 ICT4SM Lab, École Polytechnique Fédérale de Lausanne, 1020 Lausanne, Switzerland; \\ dimitris.kiritsis@epfl.ch \\ 3 Department of Engineering and Architecture, HSLU Lucerne University of Applied Sciences and Arts, \\ 6048 Horw, Switzerland; simon.zuest@hslu.ch (S.Z.); shaun.west@hslu.ch (S.W.); oliver.stoll@hslu.ch (O.S.) \\ * Correspondence: juerg.meierhofer@zhaw.ch (J.M.); jinzhi.lu@epfl.ch (J.L.)
}

Citation: Meierhofer, J.; Schweiger, L.; Lu, J.; Züst, S.; West, S.; Stoll, O.; Kiritsis, D. Digital Twin-Enabled Decision Support Services in Industrial Ecosystems. Appl. Sci. 2021, 11, 11418. https://doi.org/10.3390/ app112311418

Academic Editor: Radu Godina

Received: 2 November 2021

Accepted: 26 November 2021

Published: 2 December 2021

Publisher's Note: MDPI stays neutral with regard to jurisdictional claims in published maps and institutional affiliations.

Copyright: (c) 2021 by the authors. Licensee MDPI, Basel, Switzerland. This article is an open access article distributed under the terms and conditions of the Creative Commons Attribution (CC BY) license (https:// creativecommons.org/licenses/by/ $4.0 /)$.

\begin{abstract}
The goal of this paper is to further elaborate a new concept for value creation by decision support services in industrial service ecosystems using digital twins and to apply it to an extended case study. The aim of the original model was to design and integrate an architecture of digital twins derived from business needs that leveraged the potential of the synergies in the ecosystem. The conceptual framework presented in this paper extends the semantic ontology model for integrating the digital twins. For the original model, technical modeling approaches were developed and integrated into an ecosystem perspective based on a modeling of the ecosystem and the actors' decision jobs. In a service ecosystem comprising several enterprises and a multitude of actors, decision making is based on the interlinkage of the digital twins of the equipment and the processes, which is achieved by the semantic ontology model further elaborated in this paper. The implementation of the digital twin architecture is shown in the example of a manufacturing SME (small and medium-sized enterprise) case that was introduced in. The mixed semantic modeling and model-based systems engineering for this implementation is discussed in further detail in this paper. The findings of this detailed study provide a theoretical concept for implementing digital twins on the level of service ecosystems and integrating digital twins based on a unified ontology. This provides a practical blueprint to companies for developing digital twin based services in their own operations and beyond in their ecosystem.
\end{abstract}

Keywords: digital twin; smart services; data modeling; decision support; service ecosystems; model-based systems engineering; semantic modeling

\section{Introduction}

The motivation of this paper is to further elaborate on a conference paper [1], the aim of which was to explore how business decision making in complex manufacturing ecosystems can be supported by a systematic combination of digital twins. In contrast to digital models focusing on individual equipment, the digital twin models discussed in this paper need to incorporate a multitude of objects and resources, as well as business processes for services and operations. This is required to manage the performance of equipment and their operations. The decision problems generally relate to business processes in the entire lifecycle, and in this paper, they specifically relate to questions of maintenance and performance optimization of specific equipment in conjunction with the other machines in the installed base. It was elaborated in [1] that for objects and resources, their past, current, and predicted performance condition make up the set of relevant system variables driving the decisions. For the operations and service processes, this means taking into account service resources with limited availability. These are typically human 
resources for maintenance or spare equipment, for instance. While managing equipment based on its specific digital twin is adequate for the performance optimization of this individual equipment, we extend this view to a systems perspective by integrating the equipment into a system in conjunction with other relevant actors in the ecosystem. This multitude of objects impacting business decisions requires that a systematic combination of digital twins is designed that is tailored to the decision problems in the operations of manufacturing ecosystems.

By extending the conference paper [1], this paper further elaborates the model-based systems engineering perspective by developing the semantics modeling to the level of ontology classes, objects, and languages and then to AnyLogic [2] models for system verification. A special focus in the extension of [1] is put on the implementation of the system of digital twins in a simulation model in the case of an archetypal SME (small and medium-sized enterprise). In order to model the different individual twins of the entire system, a mixed model simulation approach is implemented and linked back to the semantic model.

The aim is to design and integrate an architecture of digital twins that is derived from the actors' needs and that leverages the potential of the synergies in the ecosystem and to demonstrate the feasibility of the implementation of this architecture in a technical simulation model. Therefore, the research questions are as follows:

1. How can multiple types of digital twins be systematically combined to create value for decision support in complex industrial service ecosystems?

2. How can digital twins be integrated to promote data interoperability in complex industrial service ecosystems?

\section{Literature Review}

West [3] described the need to identify business questions for different situations as a critical aspect of defining the use cases. The use case needs to consider the actors, processes, and the machines (e.g., people, processes, and things) to understand the problem and build, via the digital twin, different options that can support the decision-making processes. The digital twin provides a way to integrate the explicit and the tacit knowledge within a system [4]. This is important from the perspective of knowledge management, as information is only truly valuable when relevant to the individual actor. Therefore, a distinction is made between commoditized information and high-value information. High-value information offers the potential to solve individual or organizational issues and is a result of customization [5]. Shannon represents an engineering perspective towards information and semantics: "semantic aspects of communication are irrelevant to the engineering problem" [6]. Visualization of the decision support process, rather than just offering a number, provides the basis for a common understanding of the problem space and, in turn, supports value co-creation between actors with different perspectives. This is achieved in the case of the digital twin by putting the data into context [7]. This is in line with Marakas [8], who identified a knowledge base, a decision context, and points and interface as critical aspects of any decision support process.

The digital twin is defined as a virtual representation of a connected physical equipment that represents in realtime its static and dynamic characteristics [9]. It has the potential to add value to many applications and, in particular, to industrial processes, and thus attracts increasing interest among practitioners and scholars [10]. There is a wide range of understandings and definitions of the digital twin and its applications [11].

The research streams of industrial services, data-driven services, and digital twins converge in the field of decision support services. The digital twin can be considered as an implementation of data-driven services that enable the exploration of scenarios and alternatives and thus support decision making in business environments [12]. Therefore, changing the perspective and conceptualizing the digital twin as an approach for value creation from a service perspective is a promising new research direction. Decision Support Systems (DSS) are information systems that help users in their decision activities, which 
can extend to highly automated decision making. The steps of the decision making process are described in [13-17] and can be simplified to: 1. Describe the set of possible actions or alternatives. 2. Evaluate these actions. 3. Select the preferred action. Step 2 of the simplified decision process points to the application of the digital twin, in particular digital twin-based simulation, which allows users to explore the variants described in step 1 and evaluate their consequences. This is supported by [17], which states that simulation is a very common decision support instrument, in particular for decisions in maintenance or capacity planning in product-service-systems, for instance.

Nonaka talks about knowledge building being a creative cognitive process, which relates concepts far apart in an individual's memory accessible through metaphors [18]. They claim that tacit knowledge can be "transformed" into communicative knowledge by recognizing contradictions through metaphor and resolving them through analogy [19]. In addition, others emphasize that transformation is not a reproduction but rather a dynamic process of building a relationship between the individual and the state of "knowing" [20,21]. For this reason, knowledge is agile and a changing object, and using the correct information at the right time enables the development of knowledge. Therefore, decision support services must support the different perspectives of the business, technology, and operations. Zeng [22], described the application of digital technologies (i.e., digital twins and expert systems) to support decision making. Reviewing in-depth studies within the research field, Agnusdei et al. [23] observed that digital twins hold information from multiple sources, continuously updating, in order to forecast current and future conditions of the physical counterpart and to enhance decision making.

According to [15], the traditional concept of decision making has to be differentiated from the knowledge-based conception, which-according to "Service-Dominant Logic" (S-D Logic) terms [24] -integrates knowledge resources other than pure data from the systems. The approach discussed in this paper is based on the concept of S-D Logic. With the transition from products to services, the economy moves from the concept of "Goods-Dominant Logic" (G-D Logic) to S-D Logic. In S-D Logic, service is considered the fundamental purpose of economic exchange. The focus of value creation is moved from the manufacturer as creator to co-creation through customer interaction [25], i.e., the concept of industrial companies as service providers has emerged [26]. Value is created by actors integrating resources to create new so-called operant resources which create benefit for other actors [27]. The support for decision making can be considered from this perspective as a way of creating benefit for the decision maker. The resources integrated are: (a) the expert knowledge of the human actors in the system, (b) the data created by the actors, in particular by the equipment, people, and processes that are to be managed, (c) the analytics applied to these data, and (d) the decision-making process based on the integration of all these resources. This process of integrating knowledge and data from their raw formats up to supporting decisions is based on [15]. The structure is comparable to the DIKW (data-information-knowledge-wisdom) scheme based on [28]. S-D Logic states that operant resources-e.g., knowledge and skills-are the fundamental source of competitive advantage for the actors in the ecosystem. Service providers apply their knowledge and skills for the benefit of another entity or the entity itself [25]. In the context of industrial services, the ability to use a digital twin based on data-based models and analytics represents an operant resource.

\section{Materials and Methods}

The conceptual framework presented in this paper builds on and extends the framework shown in [1]. The methodology builds on the case research approach described in [29], see Figure 1. In a multiple case study with ten company cases, relevant business decision challenges in the operations of industrial equipment were researched in an explorative way, as described in detail in [4]. In the "Theory Building" phase, the key structures of the decision chain were modeled as described in Section 4.1. Given this decision chain, patterns for linking individual models impacting the decision were identified and elaborated in the 
hierarchical structure discussed in Section 4.2. The hierarchical concept for modeling the digital twins and their interrelationship in an ecosystem was derived using a top-down approach from the concept of systems engineering [30] and developed by means of an incremental differentiation of the internal structure. Further developing the interlinking of the individual twins led to the concept of semantic modeling by ontology (Sections 4.3 and 4.4). Building upon the systems engineering approach, the interplay among the different digital twins was conceptualized [31]. The theory developed in this step was then tested using a case study generalized from the ten cases. As described in Section 4.5, a simulation model was developed that was logically derived from the ontology model. This generic representative model of an ecosystem comprised archetypes of actors and digital twins. The theoretical approaches modeled in this paper are applied and discussed using this model. The simulation model was used to validate the theoretically built model. Discussing the implications of the theory built and tested (Section 6) led to a list of potential extensions and refinements, which are elaborated and discussed in Section 6.

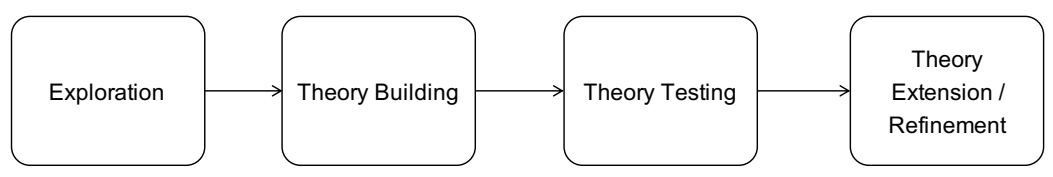

Figure 1. Methodological procedure (based on [29]).

Throughout these steps, the theory of S-D Logic [24] represents the basis for the development of the models. As described in [1], technical modeling approaches are developed and integrated into an ecosystem perspective. Against the background of service value provision, the value to be provided by the digital twins is derived from the actors decision jobs, which is relevant in order to differentiate from a technology-driven approach.

\section{Results}

This section describes the resulting technical architecture and implementation of this study. Section 4.1 discusses how the business question resulting from the decision problem is mapped to a digital twin of the equipment and the processes. Structuring the different digital twins resulting from sub-questions of the business question is elaborated in Section 4.2. Sections 4.3 and 4.4 discuss how the resulting individual digital twins are interlinked by semantic models based on ontology. The translation of the ontology model to a simulation model and its implementation with the example of the industrial case study is discussed in Section 4.5.

\subsection{Relating Business Questions to Decision Problems}

In the industrial cases where decision making is centered around operations processes, the decision chain extends from the equipment or object to the operations processes [32]. The digital twin of the equipment (see Figure 2) receives data from the real equipment and presents an indication of its performance, e.g., a health condition. For example, the data coming from the equipment may be physical sensor data, such as vibration or temperature. The digital twin model of the equipment computes the indications about the health condition based on these physical data. This indication potentially is a trigger that the current or predicted condition of the equipment is suboptimal and that it requires maintenance to be brought back to its normal condition.

However, this is raw information and it does not support decision making until it is interpreted in the context of the ecosystem. The actor managing the asset needs to decide which actions to take by trading off various resource constraints including opportunity costs for not allocating constrained resources to other assets while servicing one specific asset. In particular, this needs to take into account the time dynamics of customer challenges. For example, two machines of two different business customers with equal service contracts may require attention in the same time window. Given limited human service resources and spare parts, the manager needs to trade off which customer to serve first. This depends 
not only the nature of the technical problem, the required skill and spare parts, the traveling time, and the required effort to perform the maintenance but also on the current urgency of the reduced health condition for the customer's operations (e.g., depending on the current production shift of the customers).

The digital twin of the processes (right-hand side of Figure 2) integrates a multitude of individual digital twins representing the required information for the decisions and thus provides the required support. In real environments, this collection of digital twins cannot represent the entirety of the required information for a broad support of the decision making. Decision support based on only the available technical data would not provide the business value needed by the decision maker. Therefore, in this concept, the digital twin of the processes presents several variants of quantitatively evaluated service process actions to the decision maker, who integrates these quantitative data with with other knowledge resources (such as own management judgment) [15] — the wisdom part of the DIKW concept [28].
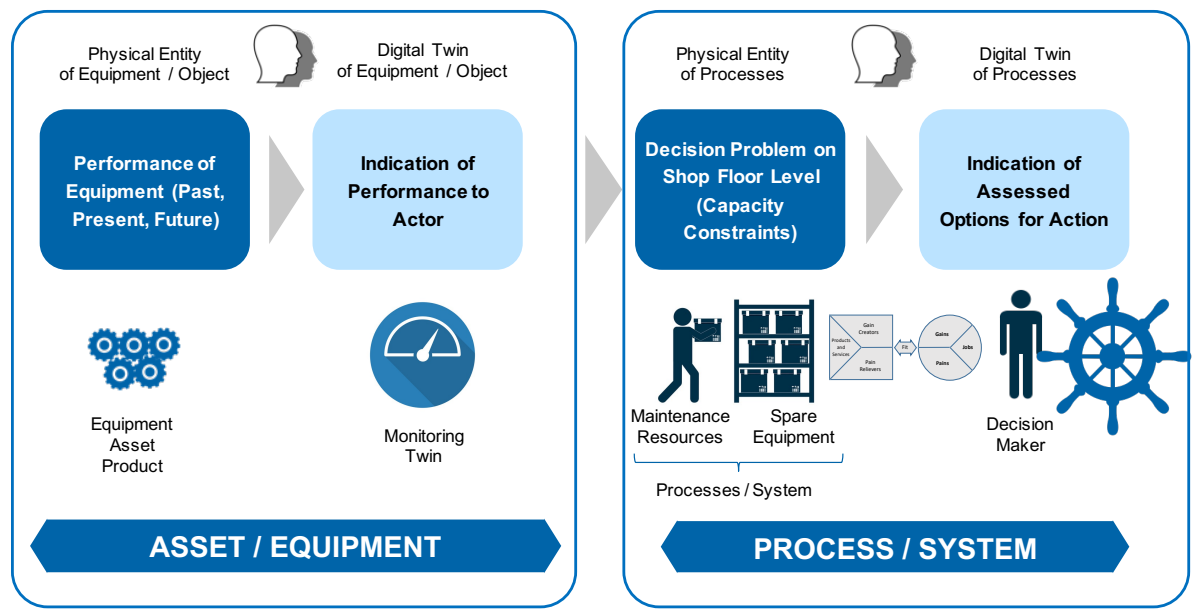

Figure 2. Digital twin of equipment vs. processes (extending on [32], terms according to [33]).

An actor managing the asset makes a decision by trading off resource constraints (Figure 3). The actor can trade off between two extremes: immediate maintenance-requiring available maintenance resources; or immediate replacement-requiring available spare equipment; or any point on the continuum between these. With the help of the process twin, the decision maker may judge whether there are spare equipment or maintenance resources available and at what cost and how to trade off the available resources among several equipment instances of the installed base.

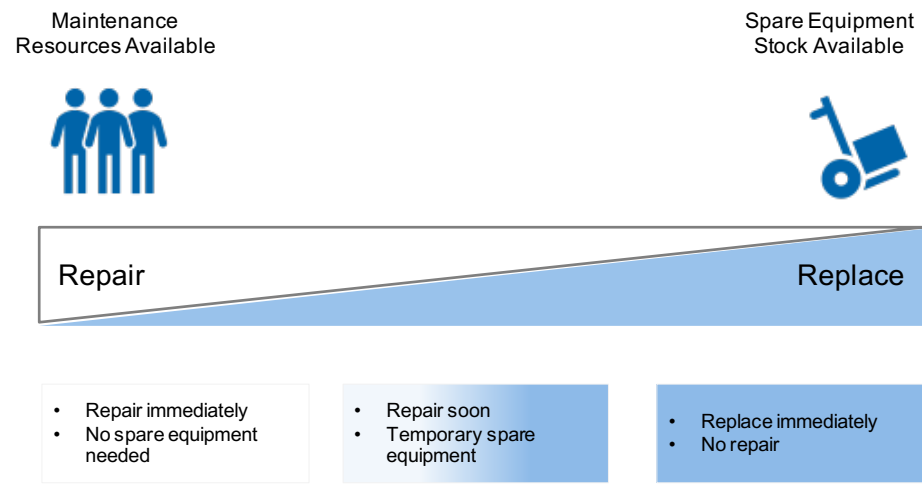

Figure 3. Tradingoff the decision between resources for maintenance and resources for spare equipment (adapted from on [32]). 


\subsection{Integrating the Domains to a Hierarchical Concept}

In a service ecosystem comprising several enterprises and a multitude of actors each having jobs to perform and decisions to be taken, usually a multitude of digital twins is required to provide decision support services to these actors. This decision making is structured in a hierarchical way following the structure of the ecosystem. The overall performance metrics can be divided into submetrics for the different hierarchical levels, e.g., a company sets a goal for the annual production output. This goal can be divided into required outputs and delivery times for the different actors in the ecosystem, which may be business units of the same company or from different companies in the ecosystem. This structure is indicated in Figure 4 by the pyramid and the KPI (key performance indicator) tree. The KPI tree includes the main KPIs, as well as the derived basic KPIs and key performance parameters (KPP) on the shop floor level as suggested by [34]. This results in several specific business challenges for different actors on different hierarchy levels, each with specific decision support needs. Depending on the information and data available, a digital twin lends itself for supporting these decisions. This will result in a digital twin landscape over the entire ecosystem with each digital twin serving a specific process owner. The individual digital twin is represented by the vertical boxes labeled "DT sequence" in Figure 4. The digital twin is thereby not limited to the hierarchy level of the process owner. It might reach over several underlying hierarchy levels depending on its scope, as well as on the system topology. A systematic development procedure consisting of six steps for the DT sequence is discussed in [35]. While isolated digital twins might have a significant value-add to the process owners, fostering the full potential requires connecting and integrating all the digital twins. In other words: an isolated twin represents untapped potential. Thus, the digital twins need to be coordinated and integrated across the entire service ecosystem. Doing so enables the prediction of the effects of decisions made on one specific hierarchy level on the performance of the processes of other hierarchy levels or ecosystem actors. This enables decisions to be made not only focusing on the local KPIs but on the overall performance of the ecosystem. Moreover, the coordinated design of the digital twins across the ecosystem draws attention to potential further digital twins that can generate additional benefits and thus close gaps in the overall hierarchical system of digital twins.

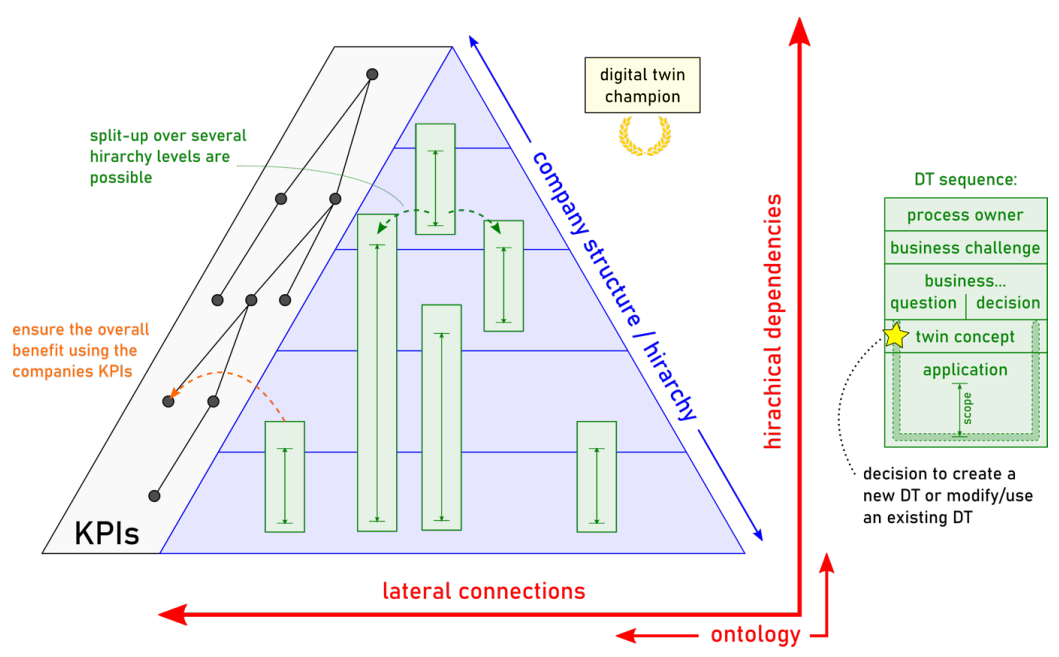

Figure 4. Hierarchical interplay between the different modeling domains.

The use of strategy deployment to cascade and translate performance metrics and KPIs is described by [36]. The approach, to be practical, requires the translation of performance metrics into metrics that are relevant for the actors at their particular level in the organization. The translation builds relevance to the actors, allowing them to take better actions based on decision making that includes an understanding of the consequences. Ref. [37] describes how strategy deployment is part of a continuous learning process and 
can change behaviors between different actors. This is important, as technical decisions in the organizations can impact the business outcomes. Negotiations are required to allow discussions between different levels within the business (with different perspectives and business languages), and the translations of metrics can support the decision making that subsequently takes place. This is particularly important as we move from one business area to another [38]. The integration of such strategy deployment within the context of a digital twin is described by [39].

The connection and integration of different digital twins requires the implementation of a new role, which we call the digital twin champion (DTC). The DTC manages and synchronizes the implementation and integration of different digital twins. Further, the knowledge transfer between different process owners regarding digital twin conceptualization, implementation, and application is organized and facilitated by the DTC. This role is of strategic importance, since the DTC has a significant effect on fostering the full potential of connected and integrated digital twins. Moreover, the DTC can identify and offer synergies between process owners with similar requirements and/or scopes on the digital twins. In the framework of S-D Logic, the DTC can be considered a task of the ecosystem orchestrator, who coordinates the collaboration through institutional arrangements.

\subsection{Ontology and Semantic Modeling for Data Integration in the Hierarchical Concept}

As discussed in Section 4.2, across the entire lifecycle, the digital twins are used by different stakeholders under several underlying hierarchy levels across different enterprises, even up to the entire industrial ecosystem. The entities and their interrelationships representing the different digital twins in an ecosystem are formalized by knowledge graph models which are developed based on the defined ontology concepts. The ontology is defined as: "an explicit, formal specification of a shared conceptualization of a domain of interest" [40], which provides a formal description of domain knowledge exploited in different ways. If a unified ontology is used for formalizing the domain-specific knowledge across the entire ecosystem [41], such knowledge can be reused efficiently without waste of R\&D costs led by non-shared knowledge. Therefore, it is important to design a unified ontology for many knowledge-intensive applications particularly for supporting digital twin integration. In order to support ontology lifecycle management, ontology engineering is proposed as a general discipline to provide methodologies and methods for building ontologies. It refers to "The set of activities that concern the ontology development and the ontology lifecycle, the methods and methodologies for building ontologies and the tool suites and languages that support them" [31]. The ontology engineering provides a guideline to define specific knowledge formalism with efficient reuse. Moreover, through such ontology, digital twins including virtual twins and their related systems can be described completely. It is the base to develop knowledge graph models for developing a seamless Information Technology (IT) infrastructure to operate with interoperability and standardization. Across the specific domains which are required to develop digital twins, ontology enables representing the nature of organizations, systems, systems engineering perspectives, system lifecycle, and virtual models of digital twins in the way of philosophy:

- Based on ontology, semantic modeling can define entities and their interrelationships, which refers to digital twin related concepts and their communications. Based on the entire ontology, entities and their topologies, digital twins, are described by the taxonomy of the defined classes and object properties [42].

- $\quad$ System models and information models based on ontology provide the ability to abstract different kinds of data in order to promote the understanding that the data are related, with good scalability.

- A semantic model refers to one type of information model describing digital twin entities and their topologies through structuring formalisms. A knowledge graph is one visualized representation of the specific semantic models using a semantic triple. Through a knowledge graph, semantic reasoning enables inference of logical consequences from a set of asserted facts or axioms. Thus, IT platforms can implement 
data analysis based on knowledge graph models through semantic query capabilities when managing the digital twins.

As shown in Figure 5, in order to manage digital twin models through developed ontology, semantic modeling enables system designers to define the meaning and context of the digital twins and the related data and to formalize the domain-specific knowledge of the physical entities of the digital twins in an abstract way. Through exploiting semantic models for digital twin management and application, several benefits are found:

- Avoiding misunderstanding of digital twins: through providing a clear, accessible, and agreed set of entities, and their relations as a trusted source and discussion, misunderstandings can easily be resolved.

- Conducting decision making through reasoning: by being machine understandable and through the usage of logic statements (rules), ontologies enable automatic reasoning and inference, which leads to automatic generation of new and implicit knowledge for decision making.

- Leveraging resources for knowledge integration: by extending and relating an application ontology to external ontological resources, the need for the repetition of the entire design process for every application domain is eliminated through manual or automatic merging and integration.

- Improving interoperability: semantic models can be used as a basis for schema matching to support systems' interoperability in a closed environment where systems, tools, and data sources have a common recognition of data types and relationships.

- Managing complexity: based on a unified semantics model, all the information related to digital twins enables IT platforms to support traceability management and consistency management.

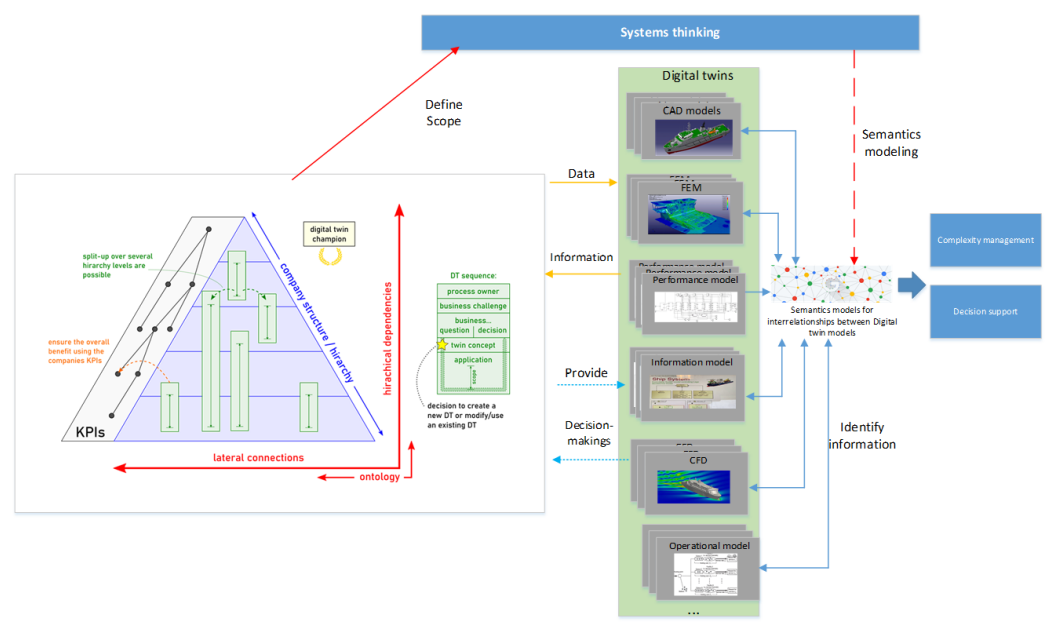

Figure 5. Semantic modeling for digital twins.

\subsection{A Semantic Modeling Approach for Integrating Digital Twins}

As shown in Figure 6, a real semantic modeling tool chain is proposed to support digital twin integration across the product lifecycle. An architecture modeling tool MetaGraph 2.0 is used to develop architecture models for managing the complexity of the digital twins using a model-based systems engineering approach [43]. Through different models, architectural views of different digital twins are described using KARMA language. Then through the GOPPRRE (Graph-object-relationship-role-point-property) ontology transformer, a GOPPRRE ontology model is generated. Then a model in the software suite AnyLogic is generated from the ontology model using a model transformer. Through this approach, the complexity of digital twins is formalized through a unified formalism and simulation models can be implemented automatically through ontology models. 


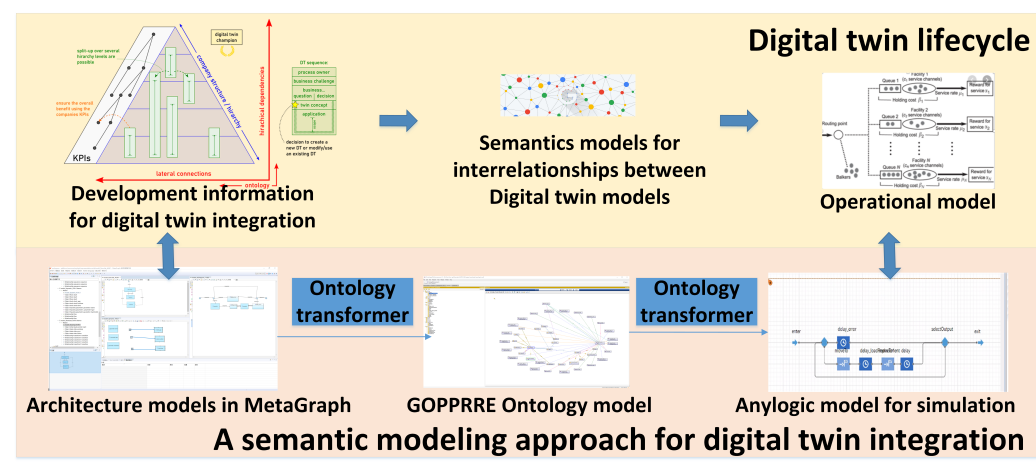

Figure 6. Ontology models for digital twins integration.

The GOPPRRE ontology refers to a unified MBSE (model-based systems engineering) ontology based on a basic MBSE formalism developed in [43]. The ontology entities and their topology were developed based on a M0-M3 modeling framework whose class and object properties are shown in Table 1. The ontology entity enables the representation of a model structure as shown in Figure 7. From the model topology, the graph individual is defined as a model to represent model compositions and the connections between them. Within one graph individual (model structure), object individuals are defined to represent model compositions. Relationship individuals are defined to represent connections between object individuals. Each relationship individual has two role individuals representing the two ends of the relationship individual. The point individuals are defined as the port for each object individual. In order to formalize the connection between object individual1 and object individual 2 through relationship individual1, two connector individuals are defined to bind role individual, relationship relationship individual, and point individual. All the non-property individuals have their own property individuals to define their attributes.

Table 1. Ontology class and object property.

\begin{tabular}{|c|c|}
\hline \multicolumn{2}{|l|}{ Class } \\
\hline connector & $\begin{array}{l}\text { its subclass defines the connection rule between one role, relationship, and } \\
\text { object in one end of each connection. }\end{array}$ \\
\hline graph & its subclass refers to a metamodel representing model topology. \\
\hline object & its subclass refers to a metamodel representing model composition. \\
\hline relationship & $\begin{array}{l}\text { its subclass refers to a metamodel representing a connection between } \\
\text { model compositions. }\end{array}$ \\
\hline role & $\begin{array}{l}\text { its subclass refers to a metamodel representing one end of a connection } \\
\text { between model compositions }\end{array}$ \\
\hline point & $\begin{array}{l}\text { its subclass refers to a metamodel representing a point in each model } \\
\text { composition. }\end{array}$ \\
\hline property & its subclass refers to an attribute for developing other metamodels. \\
\hline language & its subclass refers to a language constructed by other metamodels. \\
\hline \multicolumn{2}{|c|}{ Object property } \\
\hline binding & $\begin{array}{l}\text { Defines the connector individuals between point individual or object } \\
\text { individual and role individual in connector individuals. }\end{array}$ \\
\hline has Property & $\begin{array}{l}\text { Represents the non-property metamodel individuals having a property } \\
\text { individual referring to an attribute. }\end{array}$ \\
\hline including & $\begin{array}{l}\text { Defines the containment from a graph individual (model) to its } \\
\text { compositions (object individuals) and relationship individuals. }\end{array}$ \\
\hline link & Defines the relationship individual and its two role individuals. \\
\hline mapping & $\begin{array}{l}\text { Defines the decomposition and exploration between object individual and } \\
\text { graph individual. }\end{array}$ \\
\hline
\end{tabular}




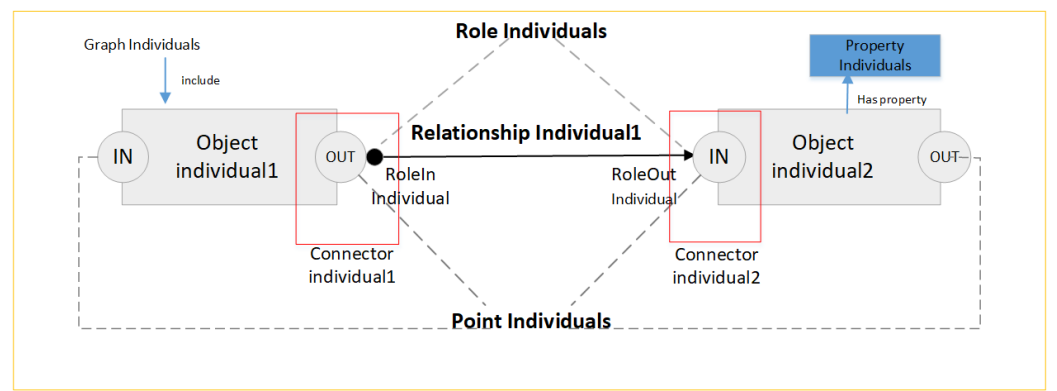

Figure 7. GOPPRRE ontology to represent model topology.

In order to realize the integration of architecture models and verification models, a model transformer was developed to transform architecture models based on KARMA language to AnyLogic models based on XML. Through this model transformation based on the GOPPRRE ontology, architecture models are transformed to AnyLogic models for verifying the performance of the process twin automatically as shown in Figure 8. In this paper, KARMA language is used to develop thearchitecture model for system architecture development as shown in Figure 9A. Through the GOPPRRE ontology generator, the GOPPRRE ontology model (Figure 9B) is generated from the architecture models.

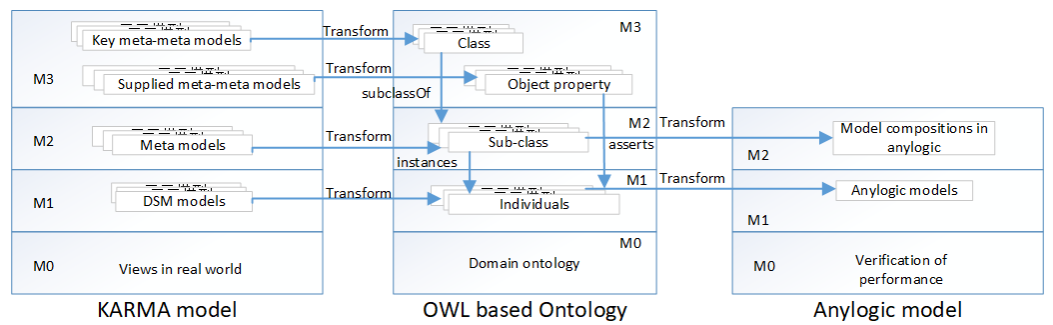

Figure 8. From architecture model to AnyLogic model.

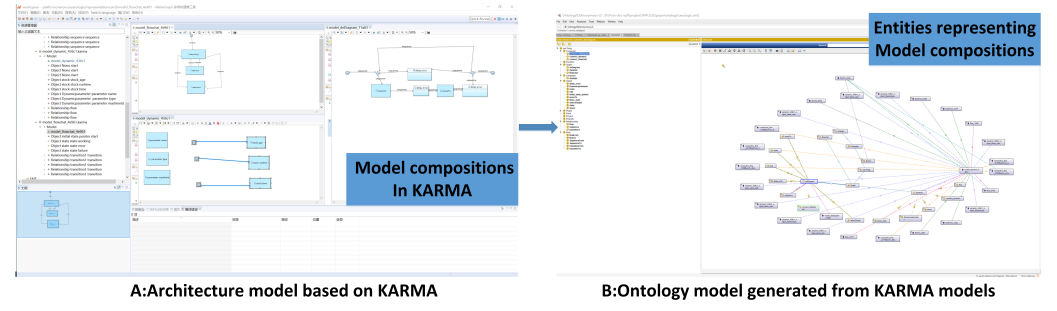

Figure 9. KARMA language to support architecture development and ontology model generated from the KARMA model.

From this section, we find a model-based systems engineering approach based on KARMA language helps to describe the multiple views of digital twins in the industrial ecosystems. Moreover, because of the KARMA language, such architecture models can generate ontology models for transforming AnyLogic models as middleware. Based on this approach, some summarized outputs are:

- Graphic representations of architectural reviews across a hierarchical interplayer between different modeling domains helps stakeholders to understand the conceptual knowledge about digital twins.

- Through the proposed semantic approach, ontology provides a good solution to complexity management in the system development [44].

- Moreover, the proposed ontology can represent the architecture models in MetaGraph 2.0 [45] and transform the ontology models into AnyLogic models as middleware. Through this semantic modeling approach, model interoperability is promoted. 


\subsection{Multi-Agent Simulation Supporting a Process Twin Construction for SMEs}

From the findings of ten case studies with companies from different industries as discussed in [3,4], we created a generic application case (Section 4.5.1). Thanks to this, the various findings from the individual cases could be condensed. For this generic case, we created a mixed model approach simulation model consisting of system dynamic, agent based, and discrete event elements (Section 4.5.2). Finally, we discussed the possibility of an automatic creation of such mixed model simulations by means of an ontology model (Section 4.5.3).

\subsubsection{Case Description for Simulation}

On the basis of the findings from Section 4.1 we designed a process twin for a hypothetical Swiss manufacturing SME, a company that is in the process of servitization. The SME already has a basic understanding of its installed base in terms of behavior, maintenance history, and location. This understanding is based on collected machine data from 100 machines located in Switzerland since 2016. Based on these data, it is already able to use machine learning to detect some component failures $24 \mathrm{~h}$ before they occur. The information about the installed base and the prediction of failures in components are helpful for the operation of the service offering that the company sells to the customers. However, there are still many unanswered questions regarding service operations, especially in dealing with predicted failures in components and the appropriate response to those eminent failures. This is where the digital process twin comes into action. In this case, the digital process twin has two tasks that involve different actors from the service ecosystem of the company. On the one hand, the digital process twin must map all relevant service operations actors that have been established in Switzerland. This includes the customers where the machines are located, and the condition of the machines with their history of maintenance and errors. On the other hand, the process twin must also be able to map the service technicians, their location, and routes to and from the customers. Furthermore, the digital service twin must be able to simulate repair and maintenance processes based on the service technicians' tasks, to estimate the working times on site. Based on these fundamentals, the following business questions need to be answered for the SME with the simulation of the digital process twin:

1. Based on the current situation, what is the best response to a predicted breakdown?

2. With how many service technicians can the service level promised to the customer be met?

3. How many spare parts are needed in stock to meet the service level promised to the customer?

The list of business questions is kept concise for the purpose of comprehensibility. It can be extended at will.

\subsubsection{Mixed Model Approach}

To meet the expectations of the business case Section 4.5.1 we used a mixed model approach to create a digital twin of the service processes. The mixed model approach structure allows a logical representation of the different hierarchical levels of the digital twins. In our mixed model approach, we combine system dynamic, agent-based, and discrete event models. This combination allows us to approximate the complex real service ecosystem. Here, the goal is to display only as much information as is necessary to answer the question. This simplification reduces the time needed to create the models and thus saves costs. Thanks to the use of agent-based structures in our model, we can represent the individual actors from the ecosystem and their behavior. Within the actors, discrete-event models allow us to represent business processes and thus control the behavior of the actors. Furthermore, by using system-dynamic models, we can simulate the physical behavior of actors, for example, in machines Figure 10. 


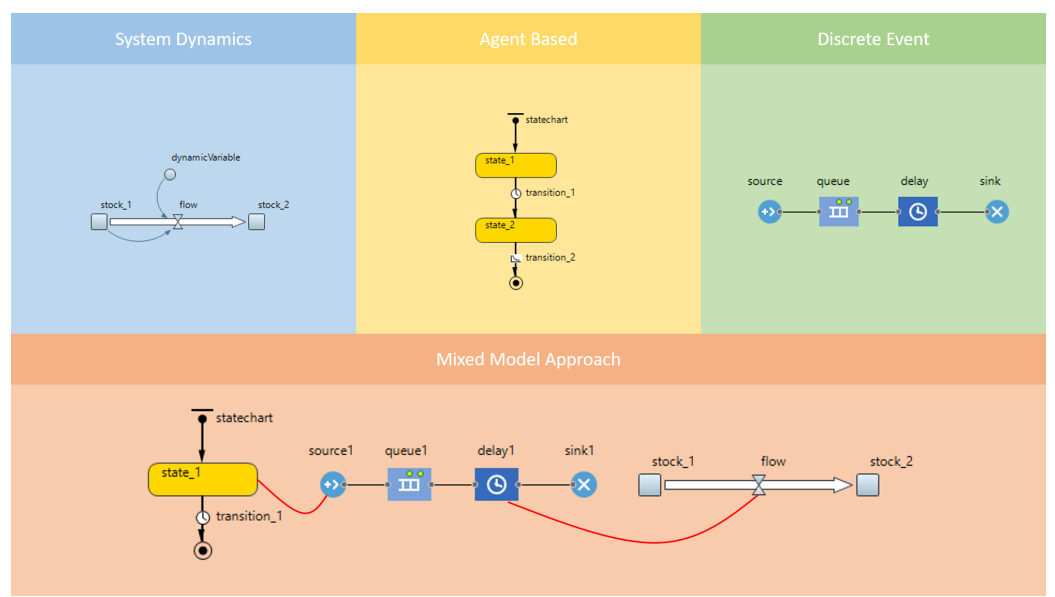

Figure 10. Combining System Dynamics, Agent Base,d and Discrete Event into a Mixed Model Approach.

For some actors in the ecosystem, agents were created, which represent them in the model. These agents are partly directly nested in each other or interact with each other via the main agent. The following agents are used in the model:

1. Main (simulation environment)

2. MillerMills (representing the company as a whole)

3. Customer

4. Machine

5. Component

6. Service Technician

7. Vehicle

Figure 11 depicts the model structure and the nesting of the different agents. Furthermore, the different simulation methods used in the different actors are indicated. This structure links the hierarchical concept shown in Figure 4 and the mixed model approach, since some elements of the simulation would also work on their own. By integrating these elements, more complex questions can be answered.

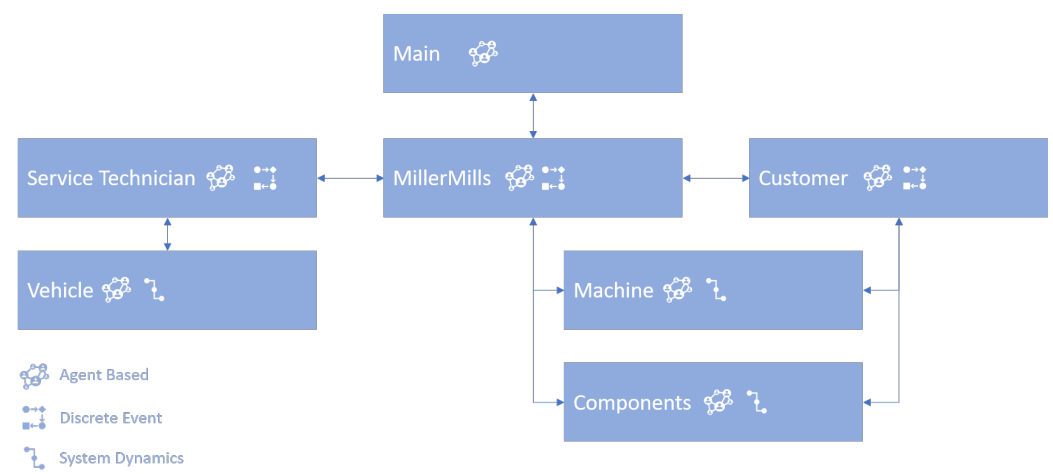

Figure 11. Structure and Types of Modeling Approaches used in Agents.

The various actors are embedded in the main agent and simulated nested in a GIS (geographic information system) environment. This GIS environment allows the user (e.g., the decision maker) to directly simulate the interaction of the service technicians, the vehicles and their routes to the customers in a reality-based environment. This allows conclusions to be drawn about travel times and possible optimization at the service technicians' starting locations. Each customer is registered as an independent agent in the GIS environment and contains the machines in operation. Each machine comprises four different components, which are simulated directly nested in the machine. MillerMills is the headquarters of the SME where the warehouse of spare parts and their production is located. The various 
agents act independently of each other and communicate their needs or inventory and availability when requested. The service technicians, for example, receive orders such as standard maintenance from the headquarters, which they have to carry out. The working hours, as well as the travel times to the customers, are simulated so that a realistic basic behavior can be created in the model. This process twin simulation model is triggered by one of following three possible triggers [46]:

1. Operator Triggers: where an operator in the system starts the process twin simulation. This triggering could be due to a machine malfunction or any other unplanned event that needs to be handled by the service department.

2. Period Triggers: recurring triggers which need to be planned. For example, yearly maintenance visits.

3. Anomaly Triggers: due to machine data and digital twins detected possible machine failures. This trigger opens the possibility to plan a suitable reaction.

Based on the simulation results, the model suggests different alternative actions. Based on this decision support, the decision maker can now plan the response in the real world in combination with its wealth of experience. Simulating those different strategic scenarios, it becomes possible to understand the consequences of different service operations decisions. Moreover, it allows certain decisions to be tested inside a safe environment. Furthermore, it becomes possible to understand the financial impacts of improvements of algorithms in error detection and different outcomes of strategies for spare part handling concerning bottlenecks or overproduction. Finally, it is also possible to adjust the number of service technicians and their training and simulate the resulting changes to the ecosystem. Figure 12 shows the model with the GIS environment including the customers, the service technicians, and MillerMills. It should also be possible to answer operational questions triggered by operator triggers in the future. For this purpose, the process twin must be enabled to analyze near-realtime data and calculate results.

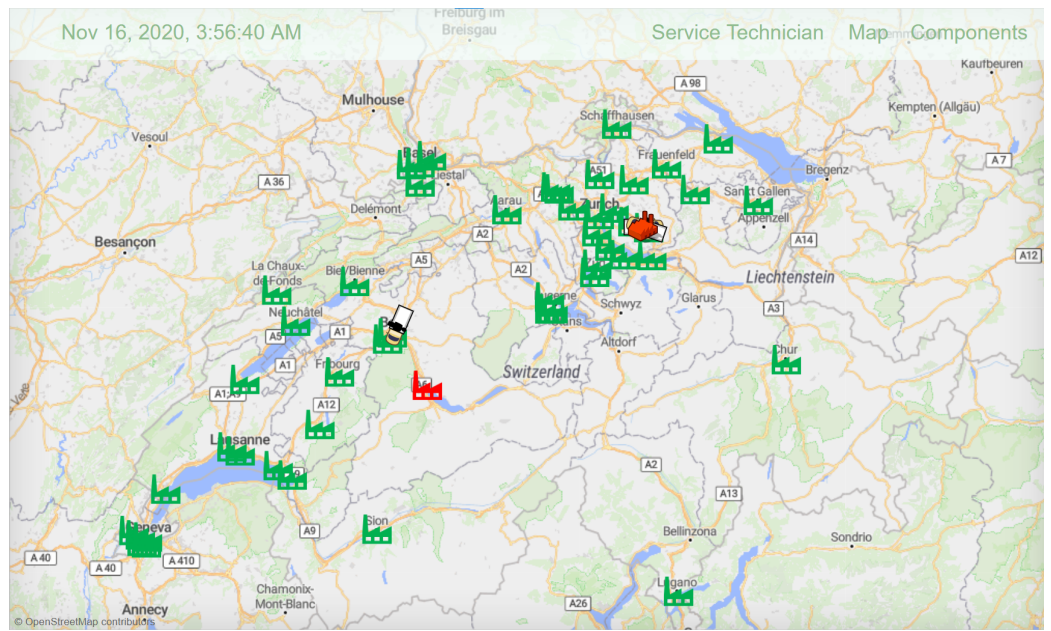

Figure 12. Main Agent of Process Twin with GIS Interface.

\subsubsection{Automatic XML Generation for Simulation Models}

In order to use the advantages of ontology described in Sections 4.3 and 4.4 and to keep the service twins up to date, it is necessary to consider automation in model creation. This could prove to be a key driver for using models in the operating environment.

As shown in Figure 8, the OWL-based Ontology needs to be transformed into XML code to be executed in the simulation software AnyLogic. Extract from the XML code of an AnyLogic simulation: 


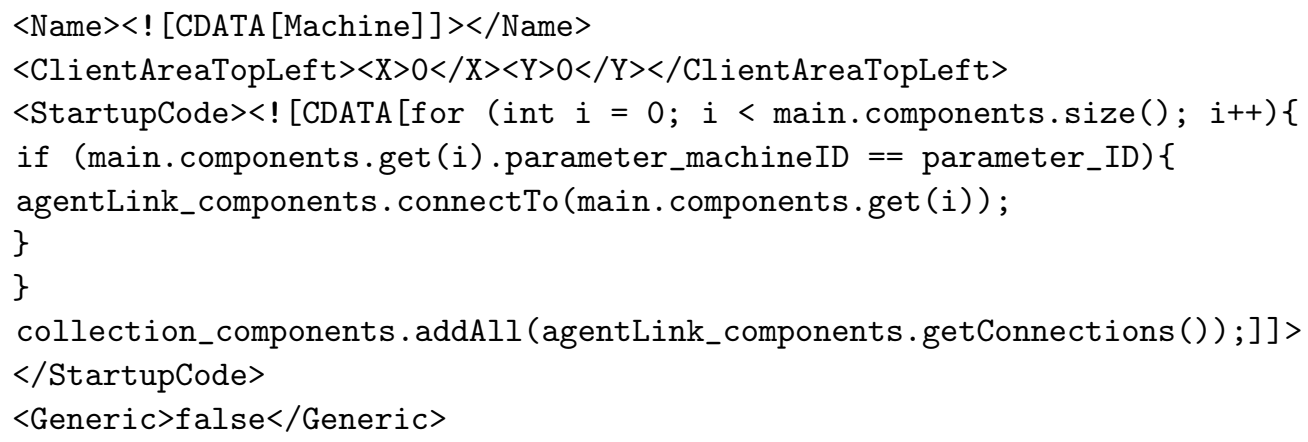

However, since the "AnyLogic project file" is not the compiled model but the source code, this must be completed in a roundabout way. One solution could be to use a java library that is able to read XML files and connect the startup code to the corresponding XML file. Based on this, one could dynamically instantiate objects inside Anylogic. This approach on building models as in Section 4.5.2 is still theoretical and was not tested in practice until now. However, there are examples of such transformations in the literature for simple models. Such a translation of OWL ontologies into simulation models would allow a consistent development of digital service twins.

\section{Theoretical and Practical Implications}

This study extended the findings of [1], which integrated the modeling approaches for digital twins and extended these to modeling on the level of service ecosystems. It provided a new concept for structuring decision problems in the operations of industrial equipment into sub-problems that can be modeled by individual digital twins on their respective level in a systematic hierarchy. This procedure resulted in a collection of elements of digital twins as shown in Figure 4. In [35], this collection is referred to as the "family of twins", together making up a digital twin concept that is derived from the business decision problems that need to be solved.

A top level business decision problem (top of the pyramid) can be broken down into sub-questions on different hierarchical levels of the ecosystem. Each question and sub-question is represented by a digital twin. Moreover, given a specific hierarchical level, there are several sub-questions and thus several twins on the same level. The interrelations between those different twins is semantically modeled by an ontology, which plays a central role in this concept.

Additionally, developing individual digital twins in the hierarchical structure and interrelating them through the concept of ontology paves the way for identifying additional digital twins in the system that were not directly derived from the a priori known decision problems but that have the potential to create additional value for specific actors in the system. For instance, when modeling a machine and its components, the ontology can show additional connections to entities or components for which an additional digital twin may improve the overall machine model. Thus, the semantic modeling by ontology substantially supports the modeling of the knowledge in the ecosystem and thus decision making.

The study also provided a concept for deriving simulation models given the hierarchical model described by the ontology. A model transformer was applied to transform the ontology model to the simulation model. Thus, the development chain from the top level business question to the simulation model implementation is represented by a consistent modeling chain.

By providing an end-to-end implementation of the new concept in a manufacturing SME case study, the theoretical concepts were applied to a specific implementation scenario shedding light on the mixed model approach for the simulation of the concept. This implementation shows how the business decision problems can be broken down into different types of system triggers and how these are modeled by specific elements in a simulation environment in a practical industrial case. 
This new modeling approach provides a blueprint for companies for developing digital twin based services in their own operations while taking into account the human and technical actors in their ecosystem that are relevant for the decision. Integrating the hierarchical concept with the concepts of ontology modeling enables leveraging the benefit of the multitude of digital twins systematically. Practical cases show that decision support for the operations of industrial equipment is required at specific stages of the lifecycle. In these cases, managers need to decide on managerial actions that have an impact not only on the equipment that is triggering the decision, but also potentially on any other equipment of the installed base. Thus, decision making is a complex task that cannot be supported by a digital twin with a narrow lens on the equipment. However, modeling an entire ecosystem through a comprehensive digital twin would be too complex and too cost intensive. Therefore, the systematic hierarchical modeling process starts with the manager's business question, developing additional digital twins only if required, and achieving their interlinking supported by semantic modeling helps solve the relevant business problems in a cost-effective way.

Similarly, three-dimensional visualization of equipment, therefore, is often not part of this concept. Instead, the individual elements provide their service to the specific users in forms that help in their context, which may be, for instance, a flag indicating which maintenance resource to send to which equipment of the installed base. Thus, applying this design approach results in a very effective and targeted digital twin model, which contains those elements and models that create value for the business decisions, and omits additional elements that are not required. This leads to cost and time savings in the development of such digital twins for enabling decision support and reduces complexity.

\section{Conclusions and Further Research}

Business processes and, in particular, service processes in industrial ecosystems extend over the lifecycle and across several entities of the ecosystem. Managerial decisions in operational processes, therefore, are complex and require taking into account a multitude of perspectives. Digital twins lend themselves to support decision making in such complex systems. However, in practical cases, the development of digital twins for complex systems may either encounter the problem of being too isolated, i.e., focusing on a too small subset of the problem space (e.g., individual assets), thus having not enough relevance for decisions impacting larger fractions of the ecosystem. Alternatively, if a more comprehensive modeling of the ecosystem is considered, the digital twin may become overly complex, resulting in too high modeling costs and potential feasibility hurdles (e.g., not enough data available across the ecosystem).

Therefore, the development of a digital twin for decision support in complex industrial ecosystems requires an approach that takes into account the entities that are relevant for supporting the decision and that leverages the interrelationships between these. The hierarchical modeling approach presented in this paper achieves this by deriving subquestions from the top level business question, defining individual digital twins per sub-question, and systematically interlinking these twins using semantic modeling based on the concept of ontologies. Through model-based system engineering, digital twins are described by KARMA language which can generate ontology models automatically. Such ontology models can be used as middleware to automatically transform them to simulation models in digital twins. Additionally, the hierarchical concept allows for a systematic perspective on the business ecosystem and for the identification of additional potential for value creation by digital twins.

The new conceptual model was developed on the basis of real cases of manufacturing companies and condensed into an application case that covers the different aspects of the ten case studies. This application shows that using the new conceptual model, different operational states in the ecosystem can be simulated, thus supporting the decision-making process for the optimal response to events. In this application, comprehensively modeling the entire ecosystem system is not required. Instead, it is sufficient to focus on the elements 
in the system that are relevant to the business decision questions, which reduces complexity and saves time and costs.

Therefore, referring to the research question formulated in the introduction, we can summarize that the paper provided an answer on how to combine and model multiple types of digital twins in complex industrial service ecosystems in order to create value for decision support. Specifically, the paper described how decision support problems can be broken down to a set of hierarchical questions, which can be translated into a hierarchical architecture of digital twins. The design and the interlinking of these twins are enabled by semantic models based on the concept of the ontology, thus promoting data interoperability in complex industrial service ecosystems. The combination of these twins is specifically targeted at supporting the decision maker's questions. The approach is effective in the sense that this specific focus on the problem means that it contains only the technical elements necessary for it.

There are a couple of limitations to this work. On one hand, the generic applicability of the framework should be validated with further cases. There is the hypothesis that resulting from this, typical patterns of combinations of digital twins will emerge. Another extension of this work may take into consideration the different phases of the equipment lifecycle. How do the decision requirements of the operations phase impact the design phase of a piece of equipment such that it lends itself to the application of the digital twin framework presented in this paper? Additionally, a future research stream will zoom in on the box named "DT sequence" in Figure 4 and focus on the question of how the underlying data and the choice of analytics and simulation methods, including algorithms, quantitatively impact the value creation for the business challenges. This in-depth analysis of the "DT sequence" aims to show which data from the business environment create value for the service providers and the service users. A clear distinction should be made between machine data and data from the ERP and CRM systems. These data are used at different points in the service process and thus generate different amounts of value for customers and providers. Based on the findings from previous research, we assume that the value of the data increases the more it lends itself to support business relevant decision making in the service domain. This hypothesis will be validated by an end-to-end approach for the use of data in service operations.

Author Contributions: Conceptualization, J.M. and S.Z.; methodology, J.M. and S.Z.; software, L.S., validation, J.M. and S.W.; formal analysis, J.M. and S.W.; investigation, J.M., L.S., J.L. and S.Z.; resources, J.M., J.L., S.W., L.S. and O.S.; data curation, L.S.; writing-original draft preparation, J.M.; writing-review and editing, J.L., S.W. and D.K.; visualization, J.M., L.S., S.Z. and J.L.; supervision, J.M.; project administration, O.S.; funding acquisition, J.M. and S.W. All authors have read and agreed to the published version of the manuscript.

Funding: This research was funded by Innosuisse (Project 35258.1 IP-SBM).

Institutional Review Board Statement: Not applicable.

Informed Consent Statement: Not applicable.

Acknowledgments: The authors would like to thank the Zurich University of Applied Sciences, the Lucerne University of Applied Sciences and Arts and the École polytechnique fédérale de Lausanne for supporting this work.

Conflicts of Interest: The authors declare no conflicts of interest. The funders had no role in the design of the study; in the collection, analyses, or interpretation of data; in the writing of the manuscript, or in the decision to publish the results. 


\section{References}

1. Meierhofer, J.; Züst, S.; Jinzhi, L.; Schweiger, L.; Kiritsis, D. Enabling decision support services in industrial ecosystems by digital twins. In Servitization: A Pathway towards a Resilient, Productive Furthermore, Sustainable Future; Aston University: Birmingham, Alabama, 2021; pp. 138-146.

2. Borshchev, A.; Karpov, Y.; Kharitonov, V. Distributed simulation of hybrid systems with AnyLogic and HLA. Future Gener. Comput. Syst. 2002, 18, 829-839. [CrossRef]

3. West, S.; Meierhofer, J.; Stoll, O.; Schweiger, L. Value propositions enabled by digital twins in the context of servitization. In Advanced Services for Sustainability and Growth; Aston University: Florence, Italy, 2020; pp. 152-160.

4. West, S.; Stoll, O.; Meierhofer, J.; Züst, S. Digital Twin Providing New Opportunities for Value Co-Creation through Supporting Decision-Making. Appl. Sci. 2021, 11, 3750. [CrossRef]

5. Dowson, R. Developing Knowledge-Based Client Relationships; Elsevier: Burlington, VT, USA, 2005.

6. Shannon, C.E. A mathematical theory of communication. ACM SIGMOBILE Mob. Comput. Commun. Rev. 2001, 5, 3-55. [CrossRef]

7. La Longa, F.; Camassi, R.; Crescimbene, M. Educational strategies to reduce risk: A choice of social responsibility. Ann. Geophys. 2012, 55. [CrossRef]

8. Marakas, G.M. Decision Support Systems in the 21st Century; Prentice Hall: Upper Saddle River, NJ, USA, 2003 ; Volume 134.

9. Romero, D.; Wuest, T.; Harik, R.; Thoben, K.D. Towards a Cyber-Physical PLM Environment: The Role of Digital Product Models, Intelligent Products, Digital Twins, Product Avatars and Digital Shadows. IFAC-PapersOnLine 2020, 53, 10911-10916. [CrossRef]

10. Barbieri, C.; West, S.; Rapaccini, M.; Meierhofer, J. Are practitioners and literature aligned about digital twin? In Proceedings of the 26th EurOMA Conference Operations Adding Value to Society, Helsinki, Finland, 17-19 June 2019;

11. Tao, F.; Qi, Q.; Liu, A.; Kusiak, A. Data-driven smart manufacturing. J. Manuf. Syst. 2018, 48, 157-169. [CrossRef]

12. Kunath, M.; Winkler, H. Integrating the Digital Twin of the manufacturing system into a decision support system for improving the order management process. In Procedia CIRP; Elsevier B.V.: Amsterdam, The Netherlands, 2018; Volume 72, pp. $225-231$. [CrossRef]

13. De Almeida, A.T.; Bohoris, G.A. Decision theory in maintenance decision making. J. Qual. Maint. Eng. 1995, 1, 39-45. [CrossRef]

14. Dong, C.S.J.; Srinivasan, A. Agent-enabled service-oriented decision support systems. Decis. Support Syst. 2013, 55, 364-373. [CrossRef]

15. W. Holsapple, C. Decisions and Knowledge. In Handbook on Decision Support Systems 1; Springer: Berlin/Heidelberg, Germany, 2008. [CrossRef]

16. Power, D.J. Decision Support Systems: A Historical Overview. In Handbook on Decision Support Systems 1: Basic Themes; Springer: Berlin/Heidelberg, Germany, 2008; pp. 121-140. [CrossRef]

17. Sala, R.; Pezzotta, G.; Pirola, F.; Huang, G.Q. Decision-support system-based service delivery in the product-service system context: Literature review and gap analysis. Procedia CIRP 2019, 83, 126-131. [CrossRef]

18. Nonaka, I.; Takeuchi, H. The Knowledge-Creating Company: How JAPANESE Companies Create the Dynamics of Innovation; Oxford University Press: Oxford, UK, 1995.

19. Nonaka, I.; Toyama, R. The knowledge-creating theory revisited: Knowledge creation as a synthesizing process. In The Essentials of Knowledge Management; Springer: Berlin/Heidelberg, Germany, 2015; pp. 95-110.

20. Bradbury, H. The Sage Handbook of Action Research; Sage: Newcastle upon Tyne, UK, 2015.

21. Shotter, J. Cultural Politics of Everyday Life: Social Constructionism, Rhetoric and Knowing of the Third Kind; University of Toronto Press: Toronto, ON, Canada, 1993.

22. Zeng, J.; Jackson, S.; Lin, I.J.; Gustafson, M.; Hoarau, E.; Mitchell, R. Operations simulation of on-demand digital print. In Proceedings of the IEEE Conference Anthology, China, 1-8 January 2013. Available online: https: / / ieeexplore.ieee.org/abstract/document/6784976?casa_token=4Lo2YXd8nMYAAAAA:fn0u6BLYgds6t_P9p6BC9fIFe7 UXtyL4tkCkSojMXKLEiEM_BVvSEoIxWA9dcc1kRIi2EYZbVQ (accessed on 25 November 2021) [CrossRef]

23. Agnusdei, G.P.; Elia, V.; Gnoni, M.G. Is Digital Twin Technology Supporting Safety Management? A Bibliometric and Systematic Review. Appl. Sci. 2021, 11, 2767.

24. Vargo, S.L.; Lusch, R.F. Evolving to a New Dominant Logic for Marketing. J. Mark. 2004, 68, 1-17.

25. Vargo, S.L.; Maglio, P.P.; Akaka, M.A. On value and value co-creation: A service systems and service logic perspective. Eur. Manag. J. 2008, 26, 145-152. [CrossRef]

26. Lay, G. Introduction. In Servitization in Industry; Lay, G., Ed.; Springer: Cham, Switzerland, 2014; pp. 1-20. [CrossRef]

27. Vargo, S.L.; Lusch, R.F. From goods to service (s): Divergences and convergences of logics. Ind. Mark. Manag. 2008, 37, 254-259. [CrossRef]

28. Rowley, J. The wisdom hierarchy: Representations of the DIKW hierarchy. J. Inf. Sci. 2007, 33, 163-180. [CrossRef]

29. Voss, C.; Tsikriktsis, N.; Frohlich, M. Case research in operations management. Int. J. Oper. Prod. Manag. 2002, 22, 195-219.

30. Züst, R. Einstieg ins Systems Engineering: Optimale, Nachhaltige Lösungen Entwickeln und Umsetzen; Orell Füssli: Zürich, Switzerland, 2004.

31. Suárez-Figueroa, M.C.; Garcia-Castro, R.; Villazón-Terrazas, B.; Gómez-Pérez, A. Essentials in ontology engineering: Methodologies, languages, and tools. In Proceedings of the 2nd Workshop Organized by the Eeb Data Models Community-CIB Conference W078-W012, Sophia Antipolis, France, 26-28 October 2011; pp. 9-21. 
32. Meierhofer, J.; West, S. Data-driven Decision Support by Digital Twins in Manufacturing. In Proceedings of the 20207 th Swiss Conference on Data Science (SDS), Luzern, Switzerland, 26 June 2020. [CrossRef]

33. Jones, D.; Snider, C.; Nassehi, A.; Yon, J.; Hicks, B. Characterising the Digital Twin: A systematic literature review. CIRP J. Manuf. Sci. Technol. 2020, 29, 36-52. [CrossRef]

34. Svantesson, T. Utilizing benchmark metrics to build and manage a strategy for maintenance improvement. Asset Manag. Maint. J. 2008. Available online: https://scholar.googleusercontent.com/scholar.bib?q=info:8cC3eB5p3LcJ: scholar.google.com/\&output=citation\&scisdr=CgU9QWZ7EITJ5CFKX5E:AAGBfm0AAAAAYaVMR5GNcuVKAk5-5p1 WX5TmXL14uWNi\&scisig=AAGBfm0AAAAAYaVMRw4pJXcpNgg6Do61Xfn8bSs7fail\&scisf=4\&ct=citation\&cd=-1\&hl=de (accessed on 25 November 2021)

35. Meierhofer, J.; West, S.; Rapaccini, M.; Barbieri, C. The Digital Twin as a Service Enabler: From the Service Ecosystem to the Simulation Model. In Exploring Service Science; Lecture Notes in Business Information Processing; Nóvoa, H., Drăgoicea, M., Kühl, N., Eds.; Springer International Publishing: Cham, Switzerland, 2020; pp. 347-359. [CrossRef]

36. Schlickel, M. Strategy Deployment in Business Units; Contributions to Management Science; Springer: Berlin/Heidelberg, Germany, 2013. [CrossRef]

37. Hines, P.; Taylor, D.; Walsh, A. The Lean journey: Have we got it wrong? Total. Qual. Manag. Bus. Excell. 2020, 31, $389-406$.

38. Yuecheng, L.; Yaoguang, H.; Peng, S.; Ruijun, Z. Research on Strategy Deployment for Manufacturing Enterprises Group. In Proceedings of the 2009 4th IEEE Conference on Industrial Electronics and Applications, Xi'an, China, 25-27 May 2009. [CrossRef]

39. Ogumerem, G.S.; Avraamidou, S.; Pistikopoulos, E.N. Computational framework for smart manufacturing via parametric optimization and control (PAROC). In Smart Manufacturing; Elsevier: Amsterdam, The Netherlands, 2020; pp. 245-259. [CrossRef]

40. Guarino, N.; Oberle, D.; Staab, S. What is an ontology? In Handbook on Ontologies; Springer: Berlin/Heidelberg, Germany, 2009; pp. 1-17.

41. Lu, J.; Wang, G.; Torngren, M. Design Ontology in a Case Study for Cosimulation in a Model-Based Systems Engineering Tool-Chain. IEEE Syst. J. 2020, 14, 1297-1308. [CrossRef]

42. Lu, J.; Zheng, X.; Gharaei, A.; Kalaboukas, K.; Kiritsis, D. Cognitive Twins for Supporting Decision-Makings of Internet of Things Systems. In Proceedings of the 5th International Conference on the Industry 4.0 Model for Advanced Manufacturing 2020, Belgrade, Serbia, 1-4 June 2020. [CrossRef]

43. Lu, J.; Ma, J.; Zheng, X.; Wang, G.; Li, H.; Kiritsis, D. Design Ontology Supporting Model-Based Systems Engineering Formalisms. IEEE Syst. J. 2021, 1-12. [CrossRef]

44. Yang, P.; Lu, J.; Feng, L.; Wu, S.; Wang, G.; Kiritsis, D. A Knowledge Management Approach Supporting Model-Based Systems Engineering. In Trends and Applications in Information Systems and Technologies; Rocha, Á., Adeli, H., Dzemyda, G., Moreira, F., Ramalho Correia, A.M., Eds.; Springe: Cham, Switzerland, 2021; pp. 581-590.

45. Lu, J.; Wang, G.; Ma, J.; Kiritsis, D.; Zhang, H.; Törngren, M. General Modeling Language to Support Model-based Systems Engineering Formalisms (Part 1). INCOSE Int. Symp. 2020, 30, 323-338. [CrossRef]

46. Tjahjono, B.; Jiang, X. Linking Symbiotic Simulation to Enterprise Systems: Framework and Applications; Institute of Electrical and Electronics Engineers Inc.: Piscataway, NJ, USA, 2015; pp. 823-834. [CrossRef] 